\title{
In Vitro Measurement of the Bone Mineral Density of the Third Metacarpal Bone by Dual Energy X-Ray Absorptiometry in Racehorses -Comparison with Single Photon Absorptiometry-
}

\author{
Masa-aki OIKAWA ${ }^{1 *}$ and Katsuhisa SHIMAZU ${ }^{2}$ \\ ${ }^{1}$ Equine Research Institute, Japan Racing Association, 27-7 Tsurumaki 5-chome, Setagaya-ku, Tokyo 154 and \\ ${ }^{2}$ the Nippon Veterinary and Animal Science University, Musashino, Tokyo 180, Japan
}

Bone mineral density $\left(B M D, \mathrm{~g} / \mathrm{cm}^{2}\right)$ in the distal portion of the third metacarpal bone (McIII) of racehorses was measured by a dual energy $x$-ray absorptiometry system (DEXA; DCS-3000, ALOKA Co, Ltd., Tokyo, Japan) in vitro, and compared with bone mineral content (BMC, g/ $\mathrm{cm}^{2}$ ) evaluated by a single photon absorptiometry system (SPA: Model 278 A, Norland Co., Fort Atkinson, U.S.A.; radioactive source was ${ }^{241} \mathrm{Am}-45 \mathrm{mCi}$ ). The BMD values measured by $D E X A$ were positively correlated with the BMC values measured by SPA, which has been used to quantitatively assess BMC with high accuracy and reproducibility in racehorses, at 0.010.0001 level with a correlative coefficient $(r)$ of 0.625-0.995 $(n=30)$. The reproducibility of values measured by DEXA shows a satisfactory coefficient value (3\%), almost same as that (2.7\%) of SPA. These results suggest the potential applications of DEXA as a quantifying instrument for the measurement of bone mass of the McIII in racehorses.

Key words: $D E X A$, bone densitometry, bone mineral density, racehorses, third metacarpal bone
J. Equine Sci.

Vol. 7, No. 4

pp. 93-96, 1996
The metacarpophalangeal joint and corresponding bones are structures which are frequently damaged in the limbs of racehorses [2]. The primary structures involved are the third metacarpal bone (McIII), first phalanx, and the proximal sesamoid bones [2]. Of these, longitudinal fractures of the distal part of McIII are suggested to occur from subchondral cancellous bone sclerosis [2]. The stiffening process was likely reflected by the increases of bone mass,i.e., bone density [6] and bone mineral content [5].

Although there are several techniques to measure bone mineral content in vivo or in vitro, photon absorptiometry using ${ }^{241}$ Americium as a source of monoenergetic $(60$ $\mathrm{KeV}$ ) photons (SPA) has been used in horses [5]. SPA has reported that the value of the McIII measured by SPA were well correlated with the weight of ashed McIII, and the reproducibility of the value was high, thus being a precise and accurate non-invasive in vitro method of quantifying bone mineral content in equine McIII [5].

Dual energy x-ray absorptiometry (DEXA), which is

This article was submitted April 26, 1996 and was accepted September 25, 1996.

*corresponding author. a relatively new photon absorptiometry, uses two energies of $\mathrm{x}$-rays as the photon source. Unlike SPA, DEXA avoids the needs for the limb to be immersed or surrounded by soft-tissue-equivalent-medium, provides images not available by SPA (so that a region of interest can be set in a fixed position while observing images and ensuring the position) and has been reported to be a quick and reliable method for determining bone mineral content [3]. The principle of the two techniques described above is to scan a bone with a narrow beam of singleenergy photons in SPA or dual-energy photons in DEXA and measure the degree of attenuation of the beam by bone, relative to its attenuation by tissue, by means of a detector system. A direct relationship is established between the number of extra photons absorbed and bone mineral content.

The objective of this study was to investigate the correlation of bone mineral content measured by SPA and that determined by DEXA for applying DEXA as the method of quantifying mineral content in vitro of the distal portion of McIII in racehorses.

Measurements of mineral contents of the McIII in racehorses was made using DEXA (DCS-3000; ALOKA 
Co., Ltd., Tokyo, Japan) and SPA (model 278A, Norland Co., Fort Atkinson, U.S.A.) [5]. The DCS-3000 uses two tightly collimated $\mathrm{x}$-ray beams of two kinds of energy levels, alternating 50 and $100 \mathrm{kVp}$. After penetrating the subject, the x-ray beams interact with the detector, in which $90 \mathrm{CdTe}$ semiconductor devices are arranged in an array, allowing computerized software analysis of beam attenuation to determine the area and composition of the subject. The use of detector with 90 semiconductor devices in an array enables the scanning time to be shortened. DEXA determines the bone mineral content in grams and the area, in $\mathrm{cm}^{2}$, of calcified tissue for the subject. Bone mineral density $\left(\mathrm{BMD} ; \mathrm{g} / \mathrm{cm}^{2}\right)$ as a measured value of mineral content is calculated by dividing the bone mineral content by the area.

SPA was used as same as mentioned in the previous report [5]. SPA indicates bone mineral content (BMC; $\mathrm{g} / \mathrm{cm}^{2}$ ), which is calculated by dividing the bone mineral content by the bone width.

As osteosclerosis of the McIII occurs severely at the distal subchondral area, BMD or BMC was measured at $1 \mathrm{~cm}$ proximal to the distal articular surface $(1 \mathrm{~cm}$ position) of the McIII, as the same position as described in a previous study [5].

A total of 30 left lower forelimbs collected from 30 racehorses of autopsies which were Thoroughbreds, 28 to 67 -months-old, weighing $428-500 \mathrm{~kg}$ (16 males, 13 females, and 1 gelding) were used for this measurement.

The BMD measurement was carried out by a DEXA in the region of interest in dorsopalmar and mediolateral directions at $1 \mathrm{~cm}$ proximal to the distal articular surface of the McIII as identified from the DEXA image (Figs. 1 and 2).

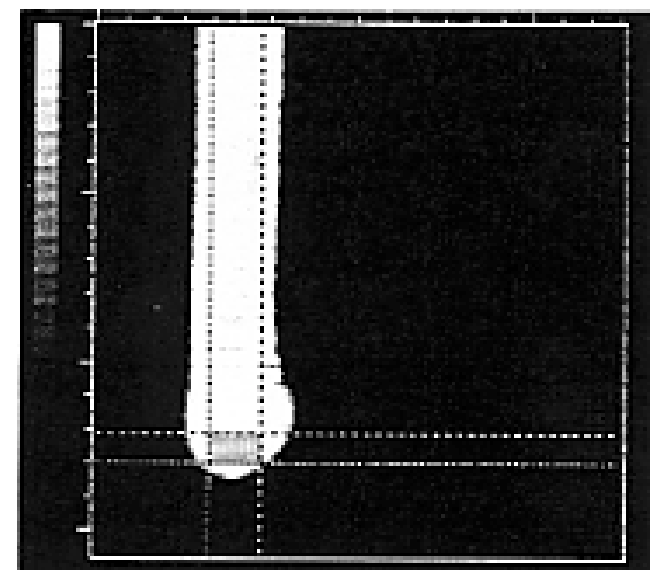

Fig. 1. DEXA image of the distal portion of McIII to show the site of bone density measurement.
The BMC measurement was carried out by a SPA in the mediolateral and dorsopalmar directions at a site 1 $\mathrm{cm}$ proximal to the distal articular surface of the McIII as described earlier (Fig. 3) [5].

To examine the reproducibility of BMD values measured by DEXA, BMD in the region of interest $\mathrm{C}$ was measured 3 times repeatedly in $15 \mathrm{McIII}$ out of the 30 forelimbs, and the coefficient of variation was calculated.

To examine usefulness of DEXA, BMD values measured by DEXA were checked by comparison with BMC values measured by SPA. A statistical analysis was made using the ANOVA-multiple method.

The values of coefficient of variation were $3 \%$ on average.

As shown in Table 1, BMD values measured by DEXA in the region of interest $\mathrm{C}$ were higher than that in the regions of interest $\mathrm{A}$ and $\mathrm{B}$. There was a highly significant positive correlation between BMD values and BMC values with a correlation coefficient $(\mathrm{r}$ ) of 0.625-0.995 $(\mathrm{p}<0.01-0.0001)$ (Table 2).

DEXA is well known to be a reliable method for determining both BMC and BMD in vitro or in vivo [1,
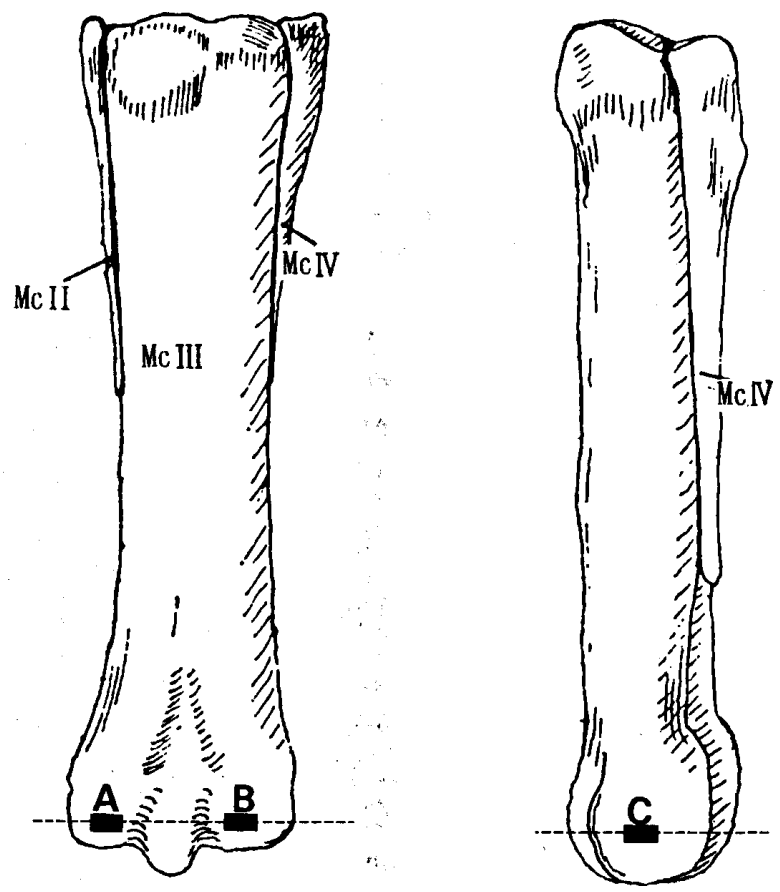

Fig. 2. Sites of bone mineral density measurement of McIII scanned by DEXA. Measurements used a $1 \times 2 \mathrm{~cm}$ area of interest, in medial (A) and lateral (B) regions from dorsopalmar direction, and in region $(\mathrm{C})$ from mediolateral direction. Dot lines indicate sites at $1 \mathrm{~cm}$ proximal to the distal articular surface of McIII. 
4]. In this study, we measured the quantitative changes in BMD for determining the mineral content and density of the McIII, as described in previous report [1, 4].

It is known that the value of BMD is influenced by the area and volume of the portion subject to measurement. In this study, we measured BMD by DEXA in portions with a certain area $(1 \times 2 \mathrm{~cm})$ but not a fixed volume. The width of the distal portion of McIII hardly differed among the racehorses. Thus, we consider that the changes of BMD among the racehorses reflects that the bone mineral content of the racehorses are different. In particular, the width of the McIII in the dorsopalmar direction varied little among the racehorses, and the correlation between BMD measured by DEXA and BMC determined by SPA happened to be the strongest between sites $\mathrm{C}$ and $\mathrm{E}$. This implies that site $\mathrm{C}$ is most suitable for detecting BMD when measured by DEXA.

The facts that BMD values evaluated by DEXA and BMC values measured by SPA at the site where bone sclerosis was the most noticeable $(1 \mathrm{~cm}$ proximal to the distal articular surface of the McIII) in racehorses showed a highly significant positive correlation, and that the value of coefficient of variation was low which was close to that $(2.7 \%)$ obtained by SPA [5] indicates that DEXA would be as precise and as accurrate an instrument as
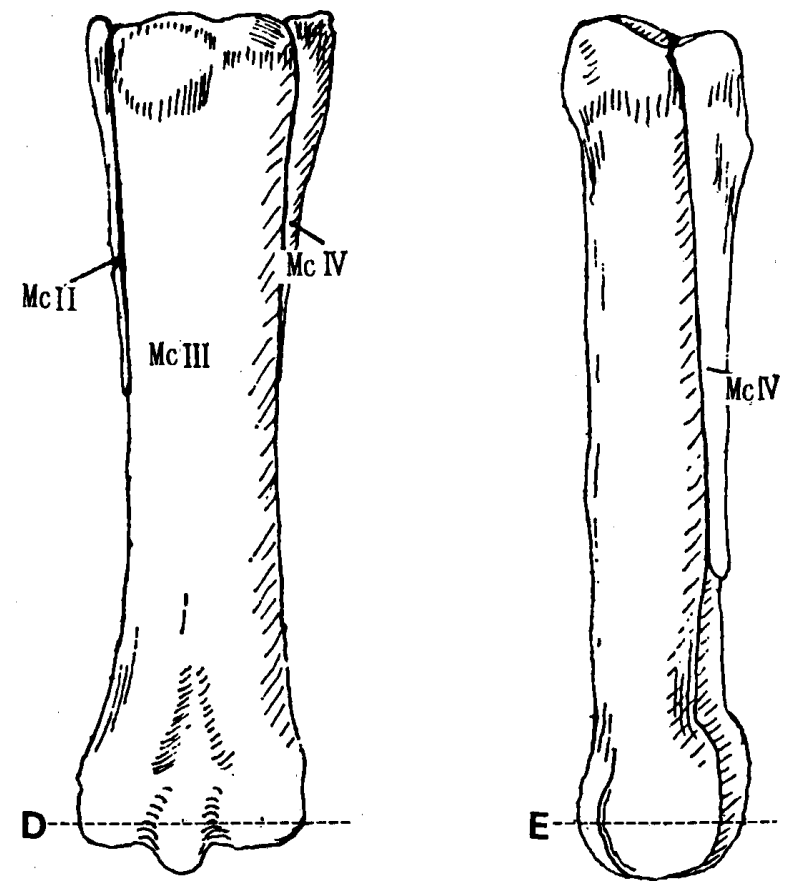

Fig. 3. Dot lines indicate regions measured by SPA in the dorsopalmar (D) and mediolateral (E) directions at a site $1 \mathrm{~cm}$ proximal to the distal articular surface of McIII.
SPA for evaluating bone sclerosis in the distal portion of the McIII of racehorses.

Table 1. BMD and BMC values of McIII in 30 Thoroughbreds

\begin{tabular}{ccc}
\hline Site $^{*}$ & BMD $^{* *}$ & BMC*** $^{* *}$ \\
\hline A & $1.791 \pm 0.185$ & \\
B & $1.798 \pm 0.267$ & \\
C & $3.514 \pm 0.386$ & \\
D & & $2.426 \pm 0.300$ \\
E & & $3.637 \pm 0.443$ \\
\hline
\end{tabular}

*: Site measured by DEXA or SPA shown in Figs. 1 or $2 .{ }^{* *}$ : Bone mineral density $\left(\mathrm{g} / \mathrm{cm}^{2}\right)$ measured by DEXA. ***: Bone mineral content $\left(\mathrm{g} / \mathrm{cm}^{2}\right)$ measured by SPA. The values for BMD and BMC are expressed as mean \pm standard deviation.

Table 2. Correlation between $\mathrm{BMD}^{1)}$ and $\mathrm{BMC}^{2)}$ in the distal portion of McIII in 30 Thoroughbreds

\begin{tabular}{ccc}
\hline Sites $^{3)}$ & $\begin{array}{c}\text { Correlation } \\
\text { coefficient }\end{array}$ & $\begin{array}{c}\text { Statistically } \\
\text { significant }\end{array}$ \\
\hline A vs B & 0.772 & $* *$ \\
A vs C & 0.723 & $* *$ \\
A vs D & 0.625 & $*$ \\
A vs E & 0.686 & $*$ \\
B vs C & 0.764 & $* *$ \\
B vs D & 0.772 & $* *$ \\
B vs E & 0.790 & $* *$ \\
C vs D & 0.908 & $* *$ \\
C vs E & 0.955 & $* *$ \\
D vs E & 0.916 & $* *$ \\
\hline
\end{tabular}

${ }^{1)}$ : Bone mineral density $\left(\mathrm{g} / \mathrm{cm}^{2}\right)$ measured by DEXA. ${ }^{2)}$ : Bone mineral content (g/ $\left.\mathrm{cm}^{2}\right)$ measured by SPA. ${ }^{3)}$ : Site measured by DEXA or SPA shown in Figs. 1 or 2. *: $\mathrm{P}<0.01$. **: $\mathrm{P}<0.001$.

Table 3. Correlation between BMD and body weight, number of races run, period on races and age

\begin{tabular}{lcc}
\hline & $\begin{array}{c}\text { Correlation } \\
\text { coefficient }\end{array}$ & $\begin{array}{c}\text { Statistical } \\
\text { significance }\end{array}$ \\
\hline C* vs Body weight $^{*}$ v No. of races run & 0.633 & $\mathrm{NS}$ \\
$\mathrm{C}^{*}$ vs & 0.785 & $\mathrm{P}<0.05$ \\
$\mathrm{C}^{*}$ vs Period on races & 0.882 & $\mathrm{P}<0.01$ \\
$\mathrm{C}^{*}$ vs Age & 0.247 & $\mathrm{NS}$ \\
\hline
\end{tabular}

*: Site measured by DEXA shown in Fig. 1. NS: Not significant. 


\section{References}

1. Eyres, K.S., Bell, M.J., and Kanis, J.H. 1993. New bone formation during leg lengthening evaluated by dual energy x-ray absorptiometry. J. Bone and Jt. Surg. 75-B: 96-106.

2. Kaneko, M., Oikawa, M., and Yoshihara, T. 1993. Pathological analysis of bone fractures in racehorses. J. Vet. Med. Sci. 55: 181-183.

3. Sartoris, D.J., and Resnick, D. 1989. Dual-energy radiographic absorptiometry for bone densitometry: current status and perspective. $\mathrm{Am}$. J. Vet. Res. 152: 241-246.

4. Tilson, D.M., Roush, J.K., McLauhlin, R.M., Toll,
Ph.W., and Richardson, D.C. 1995. Dual energy $\mathrm{x}$-ray absorptiometry (DEXA) in evaluation of the proximal femur after repair of proximal femoral physeal fractures in immature dogs. Vet. Comp. Orth. Traum. 8: 153-158.

5. Tomioka, Y., Kaneko, M., Oikawa, M., Kanemaru, T., Yoshihara, T., and Wada, R. 1985. Bone mineral content of metacarpus in racehorses by photon absorption technique: In vitro measurement. Bull. Equine Res. Inst. 22: 22-29.

6. Yoshihara, T., Kaneko, M., Oikawa, M., Wada, R., and Tomioka, Y. 1989. An application of the image analyzer to the soft radiogram of the third metacarpus in horses. Jpn. J. Vet. Sci. 51: 184-186. 